1. J. J. Sylvester, "Sur les puissances et les racines de substitutions linéaires," $C . R$. Acad. Sci. Paris, v. 94, 1882, p. 55-59.

2. A. BuchHeIM, "An extension of a theorem of Professor Sylvester's relating to matrices," Philos. Mag., v. 22, 1886, p. 173-174.

3. L. M. Milne-Thomson, The Calculus of Finite Differences, Macmillan, London, 1960.

\title{
On the Evaluation of Certain Determinants
}

\section{By Jean L. Lavoie}

1. Abstract. Using the properties of the generalized Hilbert matrix and familiar results from the theory of hypergeometric series, we evaluate the determinants of certain matrices whose general terms are known explicitly. In certain cases it is even possible to find the analytic expression for the general terms of the inverses.

2. Introduction. In this paper the matrices used are always assumed to be $n$-square and $i$ and $j$ to be positive integers such that $1 \leqq i, j \leqq n$.

The following elementary properties of determinants will be used:

(I) If $K$ multiplies all the elements in a row (column) of a determinant it multiplies the value of the determinant;

(II) the determinant of a triangular matrix is equal to the product of the $n$ terms along the main diagonal;

(III) if $A$ and $B$ are two matrices then $\operatorname{det}(A B)=\operatorname{det}(A) \operatorname{det}(B)$.

We shall also need Gauss's theorem [1, Theorem 18, p. 49]

$$
{ }_{2} F_{1}\left(\begin{array}{rr|r}
a, & b & 1
\end{array}\right)=\frac{\Gamma(c) \Gamma(c-a-b)}{\Gamma(c-a) \Gamma(c-b)},
$$

and the two following formulas, respectively from [1, example 3, p. 69] and [2, equation 8]:

$$
{ }_{2} F_{1}\left(\begin{array}{r|l}
1-a, a & \frac{1}{2}
\end{array}\right)=\frac{2^{1-c} \Gamma(1 / 2) \Gamma(c)}{\Gamma\left(\frac{c}{2}+\frac{a}{2}\right) \Gamma\left(\frac{c}{2}-\frac{a}{2}+\frac{1}{2}\right)},
$$

and

$$
{ }_{3} F_{2}\left(\begin{array}{r|r}
1-n, n+p+1, p+j & 1 \\
p+j+1, p+1 & 1
\end{array}\right)=(-1)^{n+1}(p+j) \frac{\Gamma(n) \Gamma(p+1)}{\Gamma(n+p+1)}
$$

for $j$ and $n$ positive integers, $1 \leqq j \leqq n, p \neq-1,-2, \cdots,-(2 n-1)$.

3. Preliminary Results. Let $a_{1}, a_{2}, \cdots, a_{n} ; b_{1}, b_{2}, \cdots, b_{n}$ be $2 n$ distinct but otherwise arbitrary complex numbers. Then it is well known [3, example 3 , p. 98] that the determinant of the matrix $H=\left(h_{i j}\right), \quad h_{i j}=\left(a_{i}+b_{j}\right)^{-1}$ is

$$
\operatorname{det}(H)=\frac{\prod_{r>k}^{1,2, \cdots, n}\left(a_{r}-a_{k}\right)\left(b_{r}-b_{k}\right)}{\prod_{i, j}^{1,2, \cdots, n}\left(a_{i}+b_{j}\right)} .
$$

Received December 17, 1963. 
A well-known special case of the above result is for the generalized Hilbert matrix

$$
H=\left(h_{i j}\right), \quad h_{i j}=(p+i+j-1)^{-1}, \quad p \neq-1,-2, \cdots,-(2 n-1),
$$

whose determinant is [3, p. 98-99 and 300]

$$
\text { (5) } \operatorname{det}(H)=\{1 ! 2 ! \cdots(n-1) !\}^{2} \frac{\Gamma(p+1) \Gamma(p+2) \cdots \Gamma(p+n)}{\Gamma(n+p+1) \Gamma(n+p+2) \cdots \Gamma(2 n+p)} \text {. }
$$

Using (II), let us consider the following triangular matrices and their determinants:

(6) $A=\left(a_{i j}\right), \quad a_{i j}=\frac{(-1)^{j+1} \Gamma(i)}{\Gamma(i-j+1)}, \quad \operatorname{det}(A)=(-1)^{n(n+3) / 2}\{1 ! 2 ! \cdots(n-1) !\}$,

(7) $\quad B=\left(b_{i j}\right), \quad b_{i j}=\frac{(-1)^{j+1}}{\Gamma(j) \Gamma(i-j+1)}, \quad \operatorname{det}(B)=\frac{(-1)^{n(n+3) / 2}}{\{1 ! 2 ! \cdots(n-1) !\}}$,

$$
C=\left(c_{i j}\right), \quad c_{i j}=\frac{(-1)^{j+1} \Gamma(i+j-1)}{2^{j-1} \Gamma(j) \Gamma(i-j+1)},
$$

$$
\operatorname{det}(C)=\left(-\frac{1}{2}\right)^{n(n-1) / 2} \frac{\{2 ! 4 ! \cdots(2 n-2) !\}}{\{1 ! 2 ! \cdots(n-1) !\}} .
$$

4. The Evaluation of Determinants. Our aim here is not to evaluate a long list of special determinants, but to illustrate a method by giving a few examples.

(a) Let us evaluate the determinant of the matrix

$$
E=\left(e_{i j}\right), \quad e_{i j}=\frac{1}{\Gamma(p+i+j)} .
$$

Using (6), we form the matrix product

$$
A E=\left(\alpha_{i j}\right), \quad \alpha_{i j}=\sum_{k=1}^{n} a_{i k} e_{k j} .
$$

Then from (1), we have

$$
\begin{aligned}
\alpha_{i j}=\frac{1}{\Gamma(p+j+1)}{ }_{2} F_{1}\left(\begin{array}{l|l}
1-i, 1 \\
p+j+1
\end{array}\right) & \\
& =\frac{1}{(p+i+j-1) \Gamma(p+j)} .
\end{aligned}
$$

We know $\operatorname{det}(A)$ from (6) while from (10) and (I) we have

$$
\operatorname{det}(A E)=\frac{1}{\{\Gamma(p+1) \Gamma(p+2) \cdots \Gamma(p+n)\}} \operatorname{det}(H) .
$$

$\operatorname{But} \operatorname{det}(H)$ is known from (5) and hence, using (III), we obtain

$$
\operatorname{det}(E)=\frac{\operatorname{det}(A E)}{\operatorname{det}(A)}=\frac{(-1)^{n(n+3) / 2}\{1 ! 2 ! \cdots(n-1) !\}}{\Gamma(n+p+1) \Gamma(n+p+2) \cdots \Gamma(2 n+p)} .
$$

(b) Starting with the matrix 


$$
F=\left(f_{i j}\right), \quad f_{i j}=\frac{\Gamma(i+j)}{\Gamma(p+i+j)},
$$

we form the product

$$
B F=\left(\beta_{i j}\right), \quad \beta_{i j}=\sum_{k=1}^{n} b_{i k} f_{k j}
$$

with $B$ defined by (7). Again using (1), we obtain

$$
\begin{aligned}
\beta_{i j}=\frac{\Gamma(j+1)}{\Gamma(i) \Gamma(p+j+1)}{ }_{2} F_{1}\left(\begin{array}{l|l}
1-i, j+1 \\
p+j+1
\end{array} \mid 1\right) & \\
& =\frac{\Gamma(j+1) \Gamma(p+i-1)}{\Gamma(p) \Gamma(i) \Gamma(p+i+j)}
\end{aligned}
$$

Now we know $\operatorname{det}(B)$, and from (13) and (I) we obtain

$$
\operatorname{det}(B F)=\frac{n !}{\{\Gamma(p)\}^{n}}\{\Gamma(p) \Gamma(p+1) \cdots \Gamma(p+n-1)\} \operatorname{det}(E) .
$$

But $\operatorname{det}(E)$ is known from (11), and so

$$
\begin{aligned}
\operatorname{det}(F) & =\frac{\operatorname{det}(B F)}{\operatorname{det}(B)} \\
& =\frac{n ! \Gamma(n+p)\{1 ! 2 ! \cdots(n-1) !\}^{2}\{\Gamma(p) \Gamma(p+1) \cdots \Gamma(p+n-1)\}^{2}}{\{\Gamma(p)\}^{n}\{\Gamma(p) \Gamma(p+1) \cdots \Gamma(p+2 n)\}} .
\end{aligned}
$$

(c) Again, from (8) and (9), we form the matrix product

$$
\begin{aligned}
C E & =\left(\gamma_{i j}\right), \\
\gamma_{i j} & =\frac{1}{\Gamma(p+j+1)}{ }_{2} F_{1}\left(\begin{array}{c}
1-i, i \\
p+j+1
\end{array} \mid \frac{1}{2}\right) \\
& =\frac{\Gamma(1 / 2)}{2^{p+j} \Gamma\left(\frac{p+i+j+1}{2}\right) \Gamma\left(\frac{p-i+j+2}{2}\right)}
\end{aligned}
$$

which we obtain by using (2).

But from (8) and (11) we know $\operatorname{det}(C)$ and $\operatorname{det}(E)$ so that, using (I), we easily obtain

$$
\operatorname{det}(G)=\left(\frac{2^{p+1}}{\sqrt{ } \pi}\right)^{n} \frac{\{2 ! 4 ! \cdots(2 n-2) !\}}{\Gamma(n+p+1) \Gamma(n+p+2) \cdots \Gamma(2 n+p)}
$$

where

$$
G=\left(g_{i j}\right), \quad g_{i j}=\frac{1}{\Gamma\left(\frac{p+i+j+1}{2}\right) \Gamma\left(\frac{p-i+j+2}{2}\right)} .
$$

A great number of examples can be obtained in this way since, in principle, any summation formula can be used and the triangular matrices can be chosen at will. 
Moreover, once a particular determinant is evaluated it serves as a stepping-stone to obtain other results.

5. Analytic Inversion for Some Particular Matrices. Let $D$ be a matrix whose $n$-order inverse $D^{-1}$ is known explicitly and let $S$ and $T$ be two matrices such that

$$
S T=D .
$$

Then

$$
D^{-1}=(S T)^{-1}=T^{-1} S^{-1} .
$$

Multiplying (15) successively on the left by $T$ and on the right by $S$, we obtain

$$
S^{-1}=T D^{-1}
$$

and

$$
T^{-1}=D^{-1} S \text {. }
$$

That is, the inverses of $S$ and $T$ are obtained as a product of known matrices.

Let us now consider the particular case obtained by taking for our matrix $D$ the generalized Hilbert matrix (see equation (4)), whose $n$-order inverse is well known [4] and is given by

$$
\begin{aligned}
D^{-1} & =\left(d_{i j}\right), \\
d_{i j} & =\frac{(-1)^{i+j}}{p+i+j-1} \cdot \frac{\Gamma(n+p+i) \Gamma(n+p+j)}{\Gamma(i) \Gamma(j) \Gamma(p+i) \Gamma(p+j) \Gamma(n-i+1) \Gamma(n-j+1)} .
\end{aligned}
$$

It is easy to verify, via Gauss's theorem, that the matrices

$$
\begin{aligned}
& S=\left(s_{i j}\right), \quad s_{i j}=\frac{\Gamma(p+i)}{\Gamma(p+i+j)}, \\
& T=\left(t_{i j}\right), \quad t_{i j}=\frac{(-1)^{i+1} \Gamma(j)}{\Gamma(j-i+1)},
\end{aligned}
$$

are factors of the generalized Hilbert matrix, so that from (16) and (17) it becomes possible to obtain explicit expressions for the general terms of the $n$-order inverses of $S$ and $T$.

(a) From (16), (18) and (20),

$$
S^{-1}=T D^{-1}=\left(\sigma_{i j}\right), \quad \sigma_{i j}=\sum_{k=1}^{n} t_{i k} d_{k j}
$$

and so

$$
\begin{aligned}
\sigma_{i j}= & \frac{(-1)^{i+j+1} \Gamma(n+p+j)}{\Gamma(j) \Gamma(p+j) \Gamma(n-j+1)} \\
& \cdot \sum_{k=1}^{n} \frac{(-1)^{k} \Gamma(n+p+k)}{(p+k+j-1) \Gamma(p+k) \Gamma(k-i+1) \Gamma(n-k+1)} .
\end{aligned}
$$

We notice that the first $i-1$ terms of the series are zero because of the presence 
of the factor $\Gamma(k-i+1)$ in the denominator. Hence replacing $\sum_{k=1}^{n}$ by $\sum_{k=i}^{n}$ and shifting the summation index we obtain, in the usual notation for the generalized hypergeometric series [1, p. 73],

$$
\begin{gathered}
\sigma_{i j}=\frac{(-1)^{j+1}}{p+i+j-1} \cdot \frac{\Gamma(n+p+i) \Gamma(n+p+j)}{\Gamma(j) \Gamma(p+i) \Gamma(p+j) \Gamma(n-i+1) \Gamma(n-j+1)} \\
\cdot{ }_{3} F_{2}\left(\begin{array}{rr|r}
i-n, & n+p+i, & p+i+j-1 \\
& p+i, & p+i+j
\end{array}\right) .
\end{gathered}
$$

The ${ }_{3} F_{2}(1)$ in $(21)$ can be summed in four particular cases.

For $i=1$ we use (3) and obtain

$$
\sigma_{1 j}=\frac{(-1)^{n+j} \Gamma(n+p+j)}{\Gamma(j) \Gamma(p+j) \Gamma(n-j+1)} .
$$

For $i=n$ the ${ }_{3} F_{2}(1)$ in (21) reduces to unity, and

$$
\sigma_{n j}=\frac{(-1)^{j+1} \Gamma(2 n+p) \Gamma(n+p+j-1)}{\Gamma(j) \Gamma(p+j) \Gamma(n+p) \Gamma(n-j+1)} .
$$

For $j=1$ and $j=n$ the ${ }_{3} F_{2}(1)$ in $(21)$ reduces to $a_{2} F_{1}(1)$ which can be summed by Gauss's theorem, and we obtain respectively

$$
\sigma_{i 1}=\frac{(-1)^{n+i} \Gamma(n+p+i)}{\Gamma(i) \Gamma(p+1) \Gamma(n-i+1)}
$$

and

$$
\sigma_{i n}=\frac{(-1)^{i+1} \Gamma(2 n+p) \Gamma(n+p+i-1)}{\{\Gamma(n+p)\}^{2} \Gamma(i) \Gamma(n-i+1)} .
$$

We note that it is possible to obtain a recurrence relation between $\sigma_{i+1, j}$ and $\sigma_{i, j}$ by using a known transformation of the series ${ }_{3} F_{2}(1)$.

(b) As for the matrix $T$, it is closely related to the Euler semi-matrix

$$
U=\left(u_{i j}\right), \quad u_{i j}=\frac{(-1)^{j+1}(i-1) !}{(i-j) !(j-1) !}
$$

which is its own reciprocal, and we find by inspection that

$$
T^{-1}=\left(\tau_{i j}\right), \quad \tau_{i j}=\frac{(-1)^{i+1}}{\Gamma(i) \Gamma(j-i+1)} .
$$

This result can easily be verified directly. Indeed if $r_{i j}$ is the general term of the matrix product $T T^{-1}$, then from (20) and (22) we have

$r_{i j}=\sum_{k=1}^{n} t_{i k} \tau_{k j}=\frac{(-1)^{i+1}}{\Gamma(j) \Gamma(2-i)}{ }_{2} F_{1}\left(\begin{array}{r}1-j, 1 \\ 2-i\end{array} \mid 1\right)=\frac{(-1)^{i+1}}{(j-i) \Gamma(1-i) \Gamma(j)}=\delta_{i j}$,

where as usual $\delta_{i j}=0,1$ respectively for $i \neq j, i=j$. However, from (17), $T^{-1}$ is also equal to the product $D^{-1} S$ so that (18) and (19) yield 


$$
\begin{aligned}
\tau_{i j}=\frac{(-1)^{i+1} \Gamma(n+p+1) \Gamma(n+p+i)}{\Gamma(i) \Gamma(n-i+1) \Gamma(n) \Gamma(p+j+1) \Gamma(p+i+1)} \\
\cdot{ }_{3} F_{2}\left(\begin{array}{c}
1-n, n+p+1, p+i \\
p+i+1, p+j+1
\end{array}\right) .
\end{aligned}
$$

Hence (22) and (23) imply the formula

$$
\begin{gathered}
{ }_{3} F_{2}\left(\begin{array}{c}
1-n, n+p+1, p+i \mid \\
p+i+1, p+j+1
\end{array} \mid \begin{array}{c}
1
\end{array}\right)=0 \quad \text { if } \quad 1 \leqq j<i \leqq n, \\
=\frac{\Gamma(n) \Gamma(p+i+1) \Gamma(p+j+1) \Gamma(n-i+1)}{\Gamma(n+p+1) \Gamma(n+p+i) \Gamma(1+j-i)}
\end{gathered}
$$

if $1 \leqq i \leqq j \leqq n$.

We note that for $j=1(24)$ is a special case of equation 10 of [2], if $i=j=1$ it is a special case of Saalschütz's theorem [1, Theorem 29, p. 87], while if $i=n$ or $j=n$, it reduces to Gauss's theorem.

The last relation can be proved directly by considering the formula [5, equation 2.1 , p. 238]

$$
{ }_{3} F_{2}\left(\begin{array}{r|r}
-m, a, b & 1 \\
e, f & 1
\end{array}\right)=\frac{(f-b)_{m}}{(f)_{m}}{ }_{3} F_{2}\left(\begin{array}{r}
-m, b, e-a \\
e, 1+b-f-m
\end{array} \mid 1\right) .
$$

If we write

$m=n-1, \quad a=n+p+1, \quad b=p+i, \quad e=p+j+1, \quad f=p+i+1$, we obtain

$$
\begin{aligned}
& { }_{3} F_{2}\left(\begin{array}{c|c}
1-n, n+p+1, p+i \\
p+i+1, p+j+1
\end{array}\right) \\
& =\frac{\Gamma(n) \Gamma(p+i+1)}{\Gamma(n+p+i)}{ }_{3} F_{2}\left(\begin{array}{c}
1-n, j-n, p+i \\
1-n, p+j+1
\end{array}\right) \\
& =\frac{\Gamma(n) \Gamma(p+i+1)}{\Gamma(n+p+i)}{ }_{2} F_{1}\left(\begin{array}{c}
j-n, p+i \\
p+j+1
\end{array}\right) \text { to } n \text { terms. }
\end{aligned}
$$

But for $1 \leqq j \leqq n$ the ${ }_{2} F_{1}(1)$ on the right of (25) always terminates naturally after at most $n$ terms and hence the series is complete. Summing by Gauss's theorem (1), we obtain (24).

I offer my sincere thanks to Professor F. M. Goodspeed for his kind help in this work.

Université Laval

Québec, Canada 
1. E. D. Rainville, Special Functions, Macmillan Company, New York, 1960.

2. J. L. Lavoie, "On inverses of finite segments of the generalized Hilbert matrix," $M T A C$, v. 18, 1964, p. 141-143.

3. G. Pólya \& G. Szego, Aufgaben und Lehrsätze aus der Analysis, vol. 2 (reprinted), Dover Publications, New York, 1945.

4. A. R. COLlaR, "On the reciprocal of a segment of a generalized Hilbert matrix," Proc. Cambridge Philos. Soc., v. 47, 1951, p. 11-17.

5. W. N. BAILEY, "On the sum of a terminating ${ }_{3} F_{2}(1)$," Quart. J. Math. Oxford Ser. (2) v. 4,1953 , p. $237-240$.

\title{
Conditional Least Squares Polynomial Approximation
}

\author{
By R. W. Klopfenstein
}

There are many motivations for the development of least squares polynomial approximations to sets of data. If the data is empirical, the motivation may be the smoothing out of empirical errors to obtain a representation superior in accuracy to the original data. Or if the data is in principle exact, the motivation may be to obtain a compact approximate representation for the data. In the case of data having widely variable character, it is often expedient to segment it and produce distinct polynomial representations in different ranges of the independent variable.

In many cases, it is necessary to introduce constraints on the least square approximation problem. These may occur, for example, in connection with data for which certain properties are known exactly from the underlying physical or mathematical model. They may occur also where data is to be fitted in several separate ranges and it is desired to preserve certain continuity properties from one segment of the representation to the next.

When the least square polynomials are being provided via the normal equations [1], it is often reasonably straightforward to solve the constraint equations analytically for one or more of the undetermined coefficients and insert these into the set of linear algebraic normal equations. Use of the normal equations is, however, extremely wasteful of both storage and computing time. In addition, the normal equations are notoriously poorly conditioned so that one is rarely successful in producing least square polynomials beyond the fifth or sixth degree with single precision calculations.

The least squares algorithm via orthogonal polynomials [2] is vastly superior in almost every respect. This is especially true when the Lanczos three-term recursion [3] is incorporated in the process. In this case, if the resulting polynomial is to be used solely for function evaluation purposes, it is not even necessary to produce the explicit resulting polynomial with the inherent resulting rounding problems.

It is the purpose of this note to describe a simple transformation that will permit the solution of the least squares approximation problem subject to a class of constraint conditions. This transformation results from the generalization of an approach suggested by Hamming [4]. The transformed problem is of standard least squares type without constraints and may be solved through standard algorithms for this purpose.

Received April 22, 1964. 